the antiphospholipid syndrome. J. Clin. Invest. 112:1644-1654. doi:10.1172/JCI200318817.

15. Holers, V.M., and Thurman, J.M. 2004. The alternative pathway of complement in disease: opportunities for therapeutic targeting. Mol. Immunol. 41:147-152.

16. Gigli, I., Sorvillo, J., Mecarelli-Halbwachs, L., and Leibowitch, J. 1981. Mechanism of action of the
C4 nephritic factor. Deregulation of the classical pathway of C3 convertase. J. Exp. Med. 154:1-12.

17. Botto, M., et al. 1998. Homozygous C1q deficiency causes glomerulonephritis associated with multiple apoptotic bodies. Nat. Genet. 19:56-59.

18. Bowness, P., et al. 1994. Hereditary C1q deficiency and systemic lupus erythematosus. Q. J. Med. 87:455-464.
19. Mitchell, D.A., et al. 2002. C1q deficiency and autoimmunity: the effects of genetic background on disease expression. J. Immunol. 168:2538-2543.

20. Mitchell, D.A., et al. 1999. C1q protects against the development of glomerulonephritis independently of C3 activation. J. Immunol. 162:5676-5679.

21. Botto, M., and Walport, M.J. 2002. C1q, autoimmunity and apoptosis. Immunobiology. 205:395-406.

\title{
Stat3 is required for the development of skin cancer
}

\author{
Laura Pedranzini, Andrea Leitch, and Jacqueline Bromberg
}

Memorial Sloan Kettering Cancer Center, New York, New York, USA.

\begin{abstract}
Signal transducer and activator of transcription 3 (Stat3) is a transcription factor that is constitutively activated in a variety of human malignancies, including prostate, lung, brain, breast, and squamous cell carcinomas. Inhibition of activated Stat 3 leads to decreased proliferation and apoptosis of many cancerderived cell lines, while the introduction of a constitutively activated form of Stat 3 into immortalized human breast epithelial cells and rodent fibroblasts results in cellular transformation. Collectively, these data suggest a role for Stat 3 in oncogenesis. A new study from Chan et al. (see related article beginning on page 720 ) is the first to demonstrate a requirement for Stat3 in de novo epithelial carcinogenesis in vivo. Using the two-step model of chemically induced skin carcinogenesis, the authors demonstrated that mice deficient in Stat 3 were completely resistant to skin tumor development.
\end{abstract}

Stat's (signal transducers and activators of transcription) are a family of latent transcription factors that are activated in response to many cytokines and growth factors. Stat activation is dependent upon tyrosine phosphorylation, which induces dimerization via reciprocal phosphotyrosine-src homology domain 2 (phosphotyrosine-SH2) interaction between two Stat molecules. Activated Stat's translocate to the nucleus where they bind to consensus promoter sequences of target genes and activate their transcription (1) (Figure 1). Many tyrosine kinases, including JAKs (Janus kinases), RTKs (receptor tyrosine kinases), and non-RTKs can phosphorylate Stat proteins. In normal cells, Stat tyrosine phosphorylation is transient, lasting from 30 minutes to several hours.

Nonstandard abbreviations used: DMBA, 7,12dimethylbenz[a]anthracene; Ha-ras, Harvey rat sarcoma virus oncogene; JAK, Janus kinase; LRC, label-retaining cell; RTK, receptor tyrosine kinase; SH2, src homology domain 2; Stat3, signal transducer and activator of transcription 3; TPA, 12-O-tetradecanoylphorbol-13-acetate; v-Ha-ras, Ha-ras homolog.

Conflict of interest: The authors have declared that no conflict of interest exists.

Citation for this article: J. Clin. Invest. 114:619-622 (2004). doi:10.1172/JCI200422800.
However, in numerous cancer-derived cell lines or in primary tumors, Stat proteins (in particular Stat3) are persistently tyrosine phosphorylated either as a consequence of deregulated positive effectors of Stat activation such as tyrosine kinases or negative regulators of Stat phosphorylation, e.g., phosphatases, suppressor of cytokine signaling, protein inhibitor of activated stats) (2). Inhibition of Stat 3 activity in tumor-derived cell lines by the introduction of antisense, small interfering RNA, dominant-negative Stat 3 constructs, and/or blockade of tyrosine kinases has been associated with growth arrest and apoptosis (2). Furthermore, the introduction of a constitutively activated Stat 3 molecule (Stat3C) into immortalized cell lines leads to transformation, indicating an oncogenic role for activated Stat $3(3,4)$. A possible mechanism for transformation by activated Stat 3 is the transcriptional upregulation of genes known to be involved in proliferation and apoptosis, including Bcl-xL, c-Myc, cyclin D1, Vegf, and Survivin (3, $5-8)$. In addition to its role as a transcription factor, phosphorylated Stat 3 has been described in a recent report as a component of focal adhesions (sites of cell contact with the extracellular matrix) that may contribute to the invasiveness of ovarian cancer cells (9). The in vivo role of Stat 3 in tumorigenesis has not been addressed until now. In this issue of the JCI, Chan and colleagues demonstrate in two different murine models of skin tumor development that Stat 3 is required for de novo tumorigenesis (10).

One of the best-established model systems for studying the mechanisms underlying the process of malignant transformation is the mouse skin model of multistage carcinogenesis $(11,12)$ (Figure 2). In this model, the process of skin tumor development can be subdivided into three different stages: initiation, promotion, and progression. Initiation is typically induced by the topical application of the carcinogen 7,12-dimethylbenz[a]anthracene (DMBA). Interestingly, in DMBA-treated epidermal cells, one usually finds mutations within the Harvey rat sarcoma virus oncogene (Ha-ras) gene. These mutations, however, are not sufficient to induce de novo transformation. Promotion of tumorigenesis is generated by the topical application of phorbol esters such as 12-O-tetradecanoylphorbol-13-acetate (TPA) to the skin, leading to epithelial cell proliferation with a concomitant increased expression of the ligand EGF as well as of cyclin D1, c-Jun, c-Fos, and c-Myc (13-16). TPA-treated mice form multiple benign papillomas within 10-20 weeks. Tumor progression is a spontaneous process resulting in the formation of malignant squamous carcinomas.

\section{Stat3 prevents apoptosis in the initiation phase of skin tumorigenesis}

K5Cre.Stat $3^{\mathrm{fl} / \mathrm{fl}}$ transgenic mice, whose epidermal and follicular keratinocytes lack Stat 3 , are viable and develop normally. 


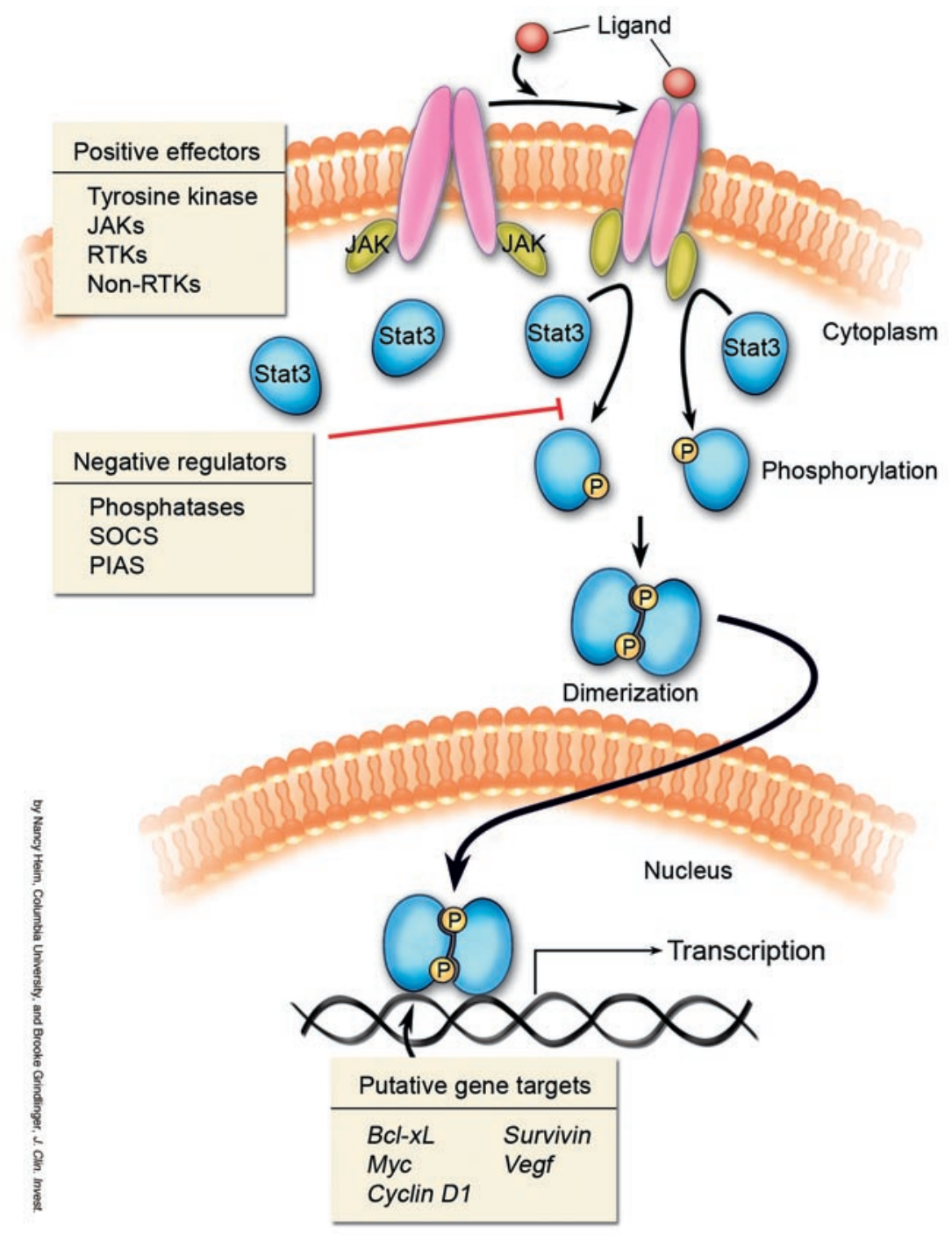

However, they have defects in hair cycle processes as well as impaired wound healing, and they develop spontaneous ulcers with age. In vitro, keratinocytes derived from these mice had no defects in proliferation, but growth factor-dependent migration was markedly impaired in contrast to control keratinocytes (17). In the study described in this issue of the JCI, Chan et al. used K5Cre.Stat $3^{\mathrm{fl} / \mathrm{fl}}$ transgenic mice to investigate the role of Stat 3 in chemically induced carcinogenesis of the skin (10). The role of Stat 3 in tumor initiation, the first step of chemically induced carcinogenesis, was addressed both in vitro, in DMBA-treated primary keratinocytes, and in vivo, by topical treatment with this mutagen. Stat 3 mutant keratinocytes underwent enhanced apoptosis following DMBA treatment, compared to control keratinocytes. Expression of Ha-ras homolog $(v$-Ha-ras $)$ into cultured primary keratinocytes in vitro was used to generate initiated keratinocytes. Upon the introduction of a Stat 3 decoy molecule (a high-affinity double-stranded DNA binding site for Stat3), the v-Ha-ras-initiated keratinocytes underwent apoptosis with a concomitant decrease in Bcl-xL levels. In general, inhibiting Stat 3 function in cancer-derived cell lines containing abundant phosphorylated Stat3 leads to apoptosis or growth arrest. In contrast, cell lines which contain low or no levels of detectable tyrosine-phosphorylated Stat 3 are relatively unaffected by Stat3 inhibitory therapies. It therefore remains unclear how Stat3 protects keratinocytes against DMBAinduced apoptosis, since DMBA does not induce tyrosine phosphorylation of Stat 3 in primary keratinocytes, nor is it likely that $\mathrm{v}$-Ha-ras-containing keratinocytes contain abundant levels of phosphorylated Stat3 $(18,19)$. Perhaps the low amounts

\section{Figure 1}

Model for Stat3 signaling. Stat3 is a transcription factor, which is activated in response to many cytokines and growth factors that bind to specific receptors. Upon ligand-receptor binding, Stat3 is recruited to the plasma membrane, where it becomes activated via phosphorylation of a tyrosine residue either directly by RTKs, such as the PDGF receptor and EGF receptor, or by non-RTKs, such as Src and JAK. Stat3 activation induces dimerization via reciprocal phosphotyrosine$\mathrm{SH} 2$ interaction between two Stat3 molecules. The Stat3 dimers then translocate to the nucleus where they bind to consensus sequences on the promoter of target genes and activate their transcription. Stat3 activation is tightly regulated by different negative regulators of phosphorylation, such as phosphatases, suppressor of cytokine signaling, and protein inhibitor of activated Stats. In many cancer-derived cell lines and primary tumors Stat3 is constitutively activated either as a consequence of deregulated signaling from positive effectors (e.g., overexpression of growth factor receptors and their ligands) or by abnormal activity of negative effectors. SOCS, suppressor of cytokine signaling; PIAS, protein inhibitor of activated Stats.

of phosphorylated Stat3 present in these cells are sufficient to drive transcription of antiapoptotic genes such as $B c l-x L$. Alternatively, nonphosphorylated Stat 3 may be playing a role as a transcription factor as has been demonstrated for Stat1 (20). There are a few notable examples where relatively low levels of phosphorylated Stat 3 are sufficient to mediate protection from growth arrest or apoptosis $(21,22)$. Thus, determination of the relative levels of phosphorylated Stat 3 required to impart a phenotype is likely to be cell-type specific and remains an important objective. It has been shown that phosphorylated Stat 1 levels are markedly enhanced in Stat 3 null hepatocytes (23). Given that Stat1 activation has been implicated in promoting growth arrest as well as apoptosis, it would be of interest to determine whether the enhanced apoptosis observed in the Stat 3 null keratinocytes correlates with increased levels of activated Stat1.

It is hypothesized that keratinocyte stem cells, which are located mostly within the bulge region of the hair follicle, are the target cells for two-stage carcinogenesis (24). Hair follicle stem cells have been identified within the label-retaining cells (LRCs), a population of cells that, following continuous administration of nucleotide analogs such as BrdU or $\left[{ }^{3} \mathrm{H}\right]$ thymidine, retains the label for a sustained period of time, indicating a very slow cycling frequency $(25,26)$. 


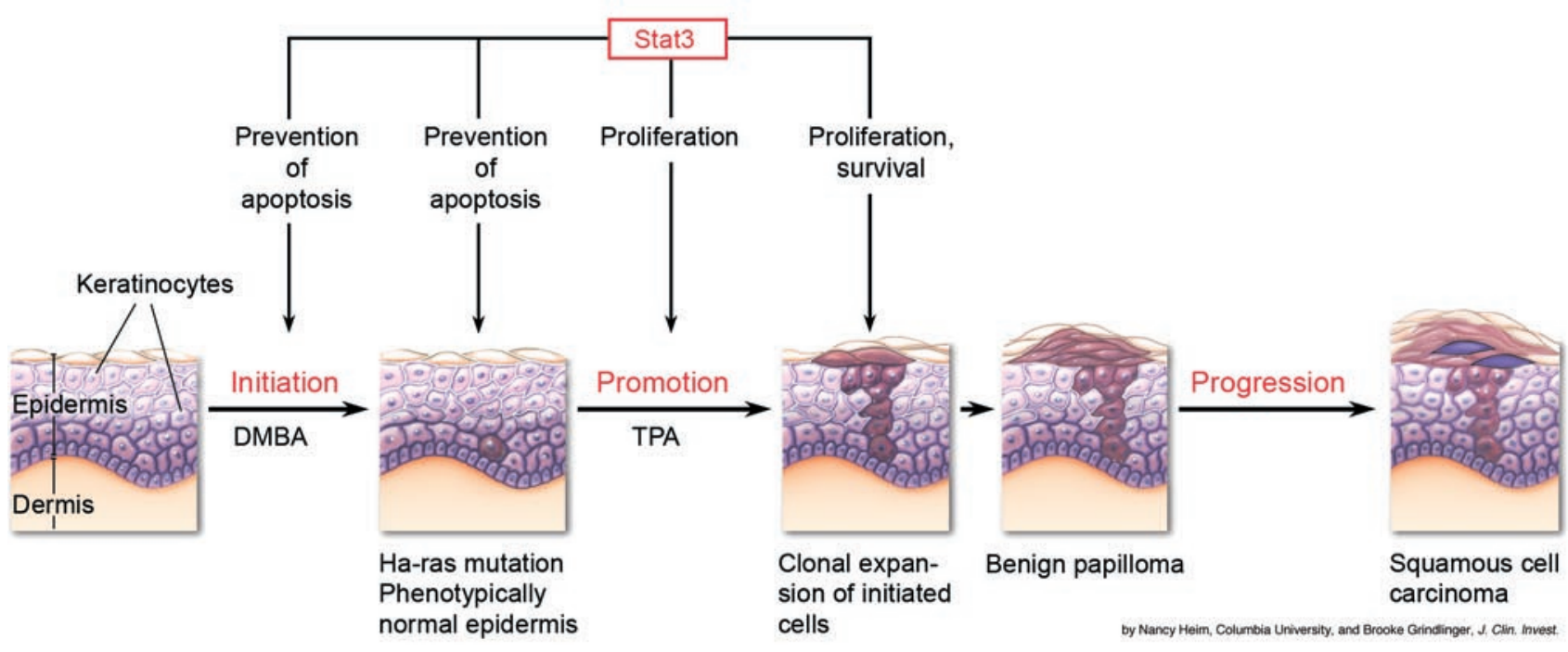

Figure 2

Two-step model of chemically induced carcinogenesis and proposed role of Stat3 in preventing tumorigenesis. Initiation is achieved by topical application of the carcinogen DMBA, which binds to DNA and typically induces mutations in the Ha-ras gene. The result of this process is the formation of an initiated cell. During this stage Stat3 protects the keratinocytes and the initiated cells from DNA damage-induced apoptosis. Promotion involves repeated application of a noncarcinogenic promoter, TPA, leading to clonal expansion of the initiated cell. This process results in the formation of multiple benign papillomas. Stat3 is essential for the proliferation of the initiated cells after TPA treatment and for the maintenance of the preoncogenic lesions. Progression is a spontaneous process characterized by the accumulation of additional genetic changes (i.e., elevated expression of genes encoding Ha-ras and cyclin D1; loss of functional p53), which accelerate the conversion of benign papillomas to malignant carcinomas. The pink cells represent initiated cells bearing Ha-ras mutations; the purple cells represent cells that have acquired multiple genetic changes.

Chan et al. (10) observed that the majority of the Stat3-deficient keratinocytes undergoing apoptosis after exposure to DMBA were located primarily within the bulge region of the hair follicle in an area adjacent to the LRC population. The authors suggest that the DMBA-sensitive cells may be keratinocyte stem cells, given their proximity to the LRCs. However, given the complete lack of overlap between the LRCs and the apoptotic cells, the cell type most sensitive to DMBA-induced apoptosis remains to be identified. The work of Chan et al. generates interesting questions regarding the mechanism(s) by which Stat 3 affords protection against apoptosis and the determination of which cell type(s) are most sensitive to the loss of Stat 3 .

\section{Stat3 is required for keratinocyte proliferation during the promotion stage of chemically induced carcinogenesis}

Tumor promotion, the second stage of chemically induced skin carcinogenesis, occurs following the topical application of TPA, leading to the proliferation of epidermal cells and the subsequent formation of benign papillomas in wild-type animals. Perhaps the most important observation made by Chan et al. (10) is that mice defi- cient in Stat3 were completely resistant to skin tumor development. Given that DMBA treatment resulted in keratinocyte apoptosis in the $\mathrm{K} 5 \mathrm{Cre}$.Stat $3^{\mathrm{fl} / \mathrm{fl}}$ animals, there was undoubtedly a decreased number of keratinocytes capable of proliferating in response to TPA. Nevertheless, the role of TPA alone (in the absence of the initiation stage) was assessed in vivo by applying TPA topically. Marked epidermal hyperproliferation and BrdU uptake was observed in wild-type animals. In contrast, significantly less proliferation was seen in the $\mathrm{K} 5 \mathrm{Cre}$.Stat $3^{\mathrm{fl} / \mathrm{fl}}$ animals. TPA induced the tyrosine phosphorylation of Stat3, perhaps in part through increased production of EGF leading to activation of the EGFR (13). An increase in some of the known putative Stat 3 targets, including cyclin D1 and $c-M y c$, was observed in the skin of wild-type animals while in the $\mathrm{K} 5 \mathrm{Cre}$.Stat $3^{\mathrm{fl} / \mathrm{fl}}$ animals a delay in the increased level of these proteins was seen. These data demonstrate that upregulation of these genes is partly dependent upon Stat 3 . The requirement of Stat 3 in tumor promotion was also examined in transgenic animals expressing v-Ha-ras targeted to keratinocytes. Topical TPA treatment of these animals led to benign papilloma formation, which was abrogated upon the introduction of a Stat 3 decoy molecule. The use of a Stat 3 decoy was first described as an inhibitor of tubular morphogenesis in cells grown in culture (27). It was subsequently shown to inhibit growth of squamous cell carcinoma-derived cell lines in vitro and more recently in vivo (28) (J. Grandis, personal communication).

The use of this molecule in vivo is indeed novel, as is the manner in which the Stat 3 decoy was administered (10). The Stat3 decoy was applied topically to the mice on a weekly basis. It is remarkable that the DNA was absorbed in sufficient quantities to exert a profound inhibitory effect on papilloma formation. Intralesional injection of the Stat 3 decoy was also effective in decreasing the size of existing papillomas. It would be of interest to know whether an increase in apoptosis was observed in the Stat3 decoy-injected lesions, as has been described for preformed B16 melanoma tumors injected with a dominant negative Stat 3 construct (29).

Decoy molecules against Stat 3 may indeed be effective therapy for cutaneous or locally advanced cancers, including skin cancers, head and neck tumors, and metastatic breast cancers involving the skin. One potential caveat with the Stat 3 decoy relates to the presumed concomitant inhi- 
bition of Stat1 activity. The decoy should bind equally well to the Stat 3 and Stat 1 dimers. Given the evidence that Stat 1 may function as a tumor suppressor as well as a promoter of apoptosis, the sequestering of the Stat 1 dimer may oppose the tumoricidal effects of sequestering Stat3 (30-32). Nevertheless, this model system of skin tumorigenesis is an ideal one for developing and testing anti-Stat 3 drugs for the prevention and treatment of skin cancer.

Address correspondence to: Jacqueline Bromberg, Memorial Sloan Kettering Cancer Center, 1275 York Avenue, New York, New York 10021, USA. Phone: (212) 639-8191; Fax: (212) 422-2231; E-mail: bromberj@mskcc.org.

1. Darnell, J.E., Jr. 1997. STATs and gene regulation. Science. 277:1630-1635.

2. Bromberg, J. 2002. Stat proteins and oncogenesis. J. Clin. Invest. 109:1139-1142. doi:10.1172/ JCI200215617.

3. Bromberg, J., et al. 1999. Stat3 as an oncogene. Cell. 98:295-303.

4. Dechow, T.N., et al. 2004. Requirement of matrix metalloproteinase- 9 for the transformation of human mammary epithelial cells by Stat3-C. Proc. Natl. Acad. Sci. U. S. A. 101:10602-10607.

5. Catlett-Falcone, R., et al. 1999. Constitutive activation of Stat 3 signaling confers resistance to apoptosis in human U266 myeloma cells. Immunity. 10:105-115.

6. Niu, G., et al. 2002. Constitutive Stat3 activity upregulates VEGF expression and tumor angiogenesis. Proc. Natl. Acad. Sci. U. S. A. 21:2000-2008.

7. Kanda, N., et al. 2004. STAT3 is constitutively activated and supports cell survival in association with survivin expression in gastric cancer cells. Oncogene. 23:4921-4929.

8. Schlette, E.J., Medeiros, L.J., Goy, A., Lai, R., and Rassidakis, G.Z. 2004. Survivin expression predicts poorer prognosis in anaplastic large-cell lymphoma. J. Clin. Oncol. 22:1682-1688.

9. Silver, D.L., Naora, H., Liu, J., Cheng, W., and Montell, D.J. 2004. Activated signal transducer and activator of transcription (STAT) 3: localization in focal adhesions and function in ovarian cancer cell motility. Cancer Res. 64:3550-3558.

10. Chan, K.S., et al. 2004. Disruption of Stat3 reveals a critical role in both the initiation and the promotion stages of epithelial carcinogenesis. J. Clin. Invest. 114:720-728. doi:10.1172/JCI200421032.

11. Angel, J.M., and DiGiovanni, J. 1999. Genetics of skin tumor promotion. Prog. Exp. Tumor Res. 35:143-157.

12. Wu, X., and Pandolfi, P.P. 2001. Mouse models for multistep tumorigenesis. Trends Cell Biol. 11:S2-S9.

13. Chan, K.S., et al. 2004. Epidermal growth factor receptor-mediated activation of Stat 3 during multistage skin carcinogenesis. Cancer Res. 64:2382-2389.

14. Robles, A.I., and Conti, C.J. 1995. Early overexpression of cyclin D1 protein in mouse skin carcinogenesis. Carcinogenesis. 16:781-786.

15. Saez, E., et al. 1995. c-fos is required for malignant progression of skin tumors. Cell. 82:721-732.

16. Hsu, J.D., et al. 1999. Suppression of the TPAinduced expression of nuclear-protooncogenes in mouse epidermis by crocetin via antioxidant activity. Anticancer Res. 19:4221-4227.

17. Sano, S., et al. 1999. Keratinocyte-specific ablation of stat 3 exhibits impaired skin remodeling, but does not affect skin morphogenesis. EMBO J. 18:4657-4668.

18. Bromberg, J.F., Horvath, C.M., Besser, D., Lathem, W.W., and Darnell, J.E., Jr. 1998. Stat3 activation is required for cellular transformation by v-src. Mol. Cell. Biol. 5:2553-2558.

19. Turkson, J., Bowman, T., Garcia, R., Caldenhoven, E., De Groot, R.P., and Jove, R. 1998. Stat3 activation by Src induces specific gene regulation and is required for cell transformation. Mol. Cell. Biol. 18:2545-2552.
20. Chatterjee-Kishore, M., Wright, K.L., Ting, J.P., and Stark, G.R. 2000. How Stat 1 mediates constitutive gene expression: a complex of unphosphorylated Stat 1 and IRF1 supports transcription of the LMP2 gene. EMBO J. 19:4111-4122.

21. Niu, G., et al. 2002. Roles of activated Src and Stat3 signaling in melanoma tumor cell growth. Oncogene. 21:7001-7010

22. Li, L., and Shaw, P.E. 2002. Autocrine-mediated activation of STAT3 correlates with cell proliferation in breast carcinoma lines. J. Biol. Chem. 277:17397-17405.

23. Li, W., Liang, X., Kellendonk, C., Poli, V., and Taub, R. 2002. STAT3 contributes to the mitogenic response of hepatocytes during liver regeneration. J. Biol. Chem. 277:28411-28417.

24. Morris, R.J. 2000. Keratinocyte stem cells: targets for cutaneous carcinogens. J. Clin. Invest. 106:3-8.

25. Morris, R.J., et al. 2004. Capturing and profiling adult hair follicle stem cells. Nat. Biotechnol. 22:411-417.

26. Tumbar, T., et al. 2004. Defining the epithelial stem cell niche in skin. Science. 303:359-363.

27. Boccaccio, C., et al. 1998. Induction of epithelial tubules by growth factor HGF depends on the STAT pathway. Nature. 391:285-288

28. Leong, P.L., et al. 2003. Targeted inhibition of Stat3 with a decoy oligonucleotide abrogates head and neck cancer cell growth. Proc. Natl. Acad. Sci. U. S. A. 100:4138-4143.

29. Niu, G., et al. 1999. Gene therapy with dominant-negative Stat3 suppresses growth of the murine melanoma B16 tumor in vivo. Cancer Res. 59:5059-5063.

30. Bromberg, J.F., Horvath, C.M., Wen, Z., Schreiber, R.D., and Darnell, J.E., Jr. 1996. Transcriptionally active Stat 1 is required for the antiproliferative effects of both IFN-a and IFN-g. Proc. Natl. Acad. Sci. U. S. A. 93:7673-7678.

31. Kaplan, D.H., et al. 1998. Demonstration of an interferon gamma-dependent tumor surveillance system in immunocompetent mice. Proc. Natl. Acad. Sci. U. S. A. 95:7556-7561.

32. Townsend, P.A., et al. 2004. STAT-1 interacts with p53 to enhance DNA damage-induced apoptosis. J. Biol. Chem. 279:5811-5820. 